\title{
Instruments to Assess the Role of the Clinical Pharmacist: A Systematic Review
}

\section{Marina Oliveira Chagas ( $\square$ marinafarm@yahoo.com.br)}

Federal University of Goias https://orcid.org/0000-0003-1619-3903

\section{Tácio de Mendonça Lima}

Federal Rural University of Rio de Janeiro Forestry Institute: Universidade Federal Rural do Rio de Janeiro Instituto de Florestas

\section{Flávio Rebustini}

University of Sao Paulo: Universidade de Sao Paulo

\section{Matias Noll}

Goiano Federal Institute: Instituto Federal Goiano

\section{Débora Penélope de Carvalho Queiroz}

$$
\text { Jatai City Council }
$$

\section{Janete Capel Hernandes}

Federal University of Goias: Universidade Federal de Goias

\section{Neuma Chaveiro}

Federal University of Goias: Universidade Federal de Goias

\section{Maria Alves Barbosa}

Federal University of Goias: Universidade Federal de Goias

\section{Celmo Celeno Porto}

Federal University of Goias

\section{Research}

Keywords: Clinical pharmacist, instruments, validation studies

Posted Date: November 6th, 2020

DOI: https://doi.org/10.21203/rs.3.rs-102462/v1

License: (c) (i) This work is licensed under a Creative Commons Attribution 4.0 International License. Read Full License 


\section{Abstract}

Background: The clinical pharmacist is an essential member of the health care team and plays an important role in health care. Therefore, knowledge regarding the instruments that evaluate the different activities of the clinical pharmacist, as well as the evaluation of the psychometric properties of these instruments, are necessary.

Methods: A literature search was performed in the PubMed and Scopus electronic databases without time and language restrictions. For the search strategy, the "pharmaceutical services", "validation studies" and "professional performance" domains were used. To assess the quality of the instruments, the five sources of validity evidence of contemporary psychometry were used, and the Joanna Briggs Institute's standardized instrument was used to assess the methodological quality of the studies. After screening 4096 articles, 32 studies were selected.

Results: It was found that the available instruments were developed or adapted from others, with variation in the methods, constructs, dimensions, and domains, as well as the psychometric properties. Most of the instruments addressed community pharmacies and evidence of content validity and internal structure was found most frequently. Conclusions: However, no validated instrument, enabling a comprehensive evaluation of all actions that pharmacists are legally qualified to perform, was found. Moreover, fragility of the instruments in concerning the psychometric properties and methodological procedures of the studies were found.

\section{Systematic Review registration: PROSPERO CRD 42018099912.}

\section{Background}

The role of the pharmacist has evolved in recent decades. There is a change in the drug supply profile, moving towards patient-centered care in order to solve pharmacotherapeutic problems and improve the quality of life of patients. The clinical pharmacist must perform activities aimed at promoting the rational use of drugs, besides identifying, solving, and preventing potential and real problems related to pharmacotherapy and other health technologies. Thus, having the practice redefined to perform actions that meet the needs of the people, family, caregivers, and the community [1].

Studies have demonstrated the expansion of this clinical role worldwide. In some countries such as Australia [2], the United States [3], and Germany [4], pharmacies are places where individuals obtain counseling on the management of the disease, review of the use of medication, prescription interventions, smoking cessation services, screening in the management of chronic diseases, and treatment of minor diseases. With this expanding practice scope, pharmacists are being recognized as key components in individualized patient care and as part of health teams [5]. In this sense, many researchers have developed instruments with the aim of evaluating this new role.

Evaluation instruments are useful and capable of presenting scientifically satisfactory results only when show robust evidence of validity. Despite the number of evaluation scales and instruments being developed, 
many are not validated and reproducible [6]. Validity was defined as "the degree to which evidence and theory support interpretations of test scores linked to the proposed uses of the tests" [7]. The evidence required for validity depends critically on the proposed interpretation of the scores and the properties of the test scores [8].

Considering this fact, this review aimed to identify and analyze the instruments that evaluate the role of the clinical pharmacist in different scenarios of pharmaceutical practice, as well as to assess the evidence of validity and the quality of the methodological procedures adopted.

\section{Methods}

This review was registered at the International Prospective Register of Systematic Reviews (PROSPERO) [9] under protocol number CRD 42018099912 and conducted in accordance with Preferred Reporting Items for Systematic reviews and Meta-Analyses (PRISMA) guidelines [10], aiming at identifying and screening eligible articles for inclusion in the study (Additional file 1).

\section{Search strategy and eligibility criteria}

The articles were searched by two independent researchers in February 2020. The searches were performed using the PubMed and Scopus databases, without time and language restrictions. The search strategy consisted of a combination of different terms and keywords from the following three domains: 1) professional, 2) instruments, and 3) professional practice. The complete search strategy is presented in Additional file 2.

The inclusion of the articles considered studies that used instruments to evaluate the performance of the clinical pharmacist, and they were: (1) studies performed in different pharmaceutical practice environments, such as community pharmacies, hospital pharmacies, outpatient pharmacies; (2) studies that evaluated the clinical activity of the pharmacist, and (3) studies with measures of validity and reliability. We excluded studies that (1) assessed other pharmaceutical activities, such as management; (2) evaluated patient satisfaction; (3) assessed the perception of other health professionals; and (4) used non-validated instruments.

\section{Study selection and data extraction}

The selection of studies was based on the results of the research in the Mendeley reference management software, with the removal of duplicates. Two reviewers (MOC and DPCQ) independently performed the selection of articles by reading the titles and abstracts. The selected texts were read in their entirety based on the established inclusion and exclusion criteria and those articles that met the criteria were included in the study. Discrepancies between reviewers were resolved by a third reviewer (MN).

Extraction of data was performed independently by the same reviewers (MOC and DPCQ) utilizing an Excel sheet. The collected information was authors, country, method, language, objectives of the study, scenario, measurement instrument, sample, instrument construct, dimensions and domains, sections and items, validity, and reliability. 


\section{Assessment procedures}

\section{Assessment of methodological quality}

The methodological quality of the studies was evaluated using standardized tools for critical evaluation of the Meta-Analysis of Statistics Assessment and Review Instrument of the Joanna Briggs Institute (JBIMAStARI) [11]. This instrument has eight questions: 1.The sample was appropriate to address the target population, 2. Criteria for inclusion in the sample clearly defined, 3. Adequate sample size, 4.Study subjects and the setting described in detail, 5.Analysis conducted with sufficient coverage of the identified sample, 6.Outcomes measured in a valid way, 7.0bjective and standard criteria for measurement, 8.Appropriate statistical analysis, The critical evaluation checklists used in this study were intended for observational studies, such as analytical cross-sectional studies (Additional file 3 ). The risk of bias was classified as high when the studies reached up to $49 \%$ of the "yes" score; moderate when they reached $50-69 \%$ of the "yes" score, and low when the studies reached more than $70 \%$ of "yes" score [12].

\section{Assessment of psychometric quality}

The assessment of the psychometric quality of methodological studies was carried out using the script of contemporary field of the state-of-the-art of psychometry $[7,8,13]$. The five sources of validity evidence based for instruments considered for analysis were: 1. Content, 2. Response process, 3. Internal structure, 4. Relations to other variables and 5. Consequences. Thus, the quality of each instrument for each study was assessed separately. They were classified according to the proposed criteria of the Cochrane Back Review Group [14] as strong (consistent positive results from multiple studies with good methodological quality or one study with excellent methodological quality), moderate (consistent positive results from multiple studies with fair methodological quality or one study with good methodological quality), limited (positive results from a study with fair methodological quality), conflicting (conflicting results from individual studies), or unknown (results from studies with poor methodological quality with an unknown level of evidence).

\section{Results}

A total of 4096 articles were found in the databases. After removing the duplicates $(n=1018)$ and including the results of the manual search $(n=4), 3082$ articles remained for reading the titles and abstracts. After sorting the titles and abstracts, 2909 articles were excluded and 173 articles remained for reading in full, applying the eligibility criteria. Of these, 32 articles met the criteria and were included in the review (Fig. 1).

\section{Characteristics of the studies}

The characteristics of the 32 studies are presented in Additional file 4. The year of publication ranged from 1994 to 2018, and the study published in 1994 was the first to be identified that used a questionnaire to investigate the involvement of community pharmacists in education and disease prevention activities. As for the language, 31 studies were published in English and one in Spanish. The sample size ranged from 69 to 4696 pharmacists. 
Regarding the country of publication, the instruments were developed in North American (11 studies: 6 in Canada and 5 in the United States), Asian (11 studies: 3 in Qatar; 1 in Pakistan; 1 in Jordan; 1 in Malaysia; 1 in China; 1 in Thailand; 1 in Saudi Arabia; 1 in Indonesia, and 1 in Kuwait), European (7 studies: 2 in United Kingdom countries; 1 in Denmark; 1 in Belgium; 1 in Spain; 1 in Serbia, and 1 comprising several European countries), African (2 studies in Sudan), and Oceania (1 study in Australia) countries.

As for the study type, 22 were classified as methodological and cross-sectional, 5 methodological studies and 5 cross-sectional studies. In methodological and cross-sectional studies, the creation of the instruments came from literature review, literature review and/or based on documents (standards, laws), literature review and experts and authors' consensus. Concerning the methodological studies that developed new instruments, the authors used qualitative (interviews/focus group) and quantitative approaches. In the five cross-sectional studies, the instruments came from adapted versions of other existing instruments.

\section{Characteristics of the instruments}

The instruments are divided into "general" or "specific" according to the approached construct. Among the general instruments, ten instruments addressing pharmaceutical care $[15,17,18,20,21,22,23,24]$ were identified and they evaluated the following: frequency in the identification and resolution of medicationrelated problems (assessment of the patient, monitoring of therapy, process documentation, and collaboration with other health professionals), attitudes in the practice of pharmaceutical care, and barriers to involvement in pharmaceutical care.

Regarding the specific instruments, they were divided into specific health/disease conditions $(n=9)$ and services/activities $(n=13)$. The instruments aimed at specific conditions evaluated the performance of the pharmacist for disease conditions [26, 27, 28, 29, 30, 31, 32, 33] such as diabetes, depression, cardiovascular diseases, pregnancy/lactation, mental illness, smoking, and chronic diseases. In these instruments, the following issues were addressed: involvement in self-care services (counseling on therapies and diseases), dispensing activities, involvement in counseling in healthy living promotion, patient education, attitudes in providing services/activities, and the main barriers identified.

Regarding the specific instruments for services/activities [34, 35, 36, 37, 39, 40, 41, 42, 43, 44, 45], the following were identified: instruments for education and health promotion, counseling in drug disposal, medication monitoring, reporting of adverse reactions, involvement in patient safety, acting on prescriptions, involvement in clinical research activities, drug therapy management service, pharmacotherapy follow-up service, immunization service, and oral health.

Regarding the location of practice, most of the studies were focused on pharmacists working in community pharmacies $(n=22)$. The others focused on hospital pharmacies $(n=4)$, different practice environments ( $n$ $=3)$; primary care pharmacies $(n=1)$; community and hospital pharmacies $(n=1)$, and community and outpatient pharmacies $(n=1)$.

In general, in relation to the structure, the instruments showed variations in the number of items (12 to 101 items). As for the sections, there was a variation between 2 and 6 sections, with 12 instruments not reporting the quantity and regarding the domains and/or dimensions only 13 instruments described and 
reported the quantities. Regarding validity, most studies presented information on content and internal structure (Table 2).

The characteristics of the 32 instruments as well as their psychometric properties are described in Additional file 4 and 5 , respectively.

\section{Quality of psychometric properties of instruments}

The instruments were assessed for the five sources of validity evidence, which correspond to the content, the response process, the internal structure, other variables, and the consequences. In the analysis, not all presented the sources of evidence described, as shown in Table 1. 
Table 1

Levels of evidence of the quality of psychometric properties of the instruments

\begin{tabular}{|c|c|c|c|c|c|c|}
\hline Instruments & Author/Year & Content & $\begin{array}{l}\text { Response } \\
\text { process }\end{array}$ & $\begin{array}{l}\text { Internal } \\
\text { structure }\end{array}$ & $\begin{array}{l}\text { Other } \\
\text { variables }\end{array}$ & Consequences \\
\hline \multicolumn{7}{|c|}{ General Instruments } \\
\hline BPCS & $\begin{array}{l}\text { Odedina FT, Segal } \\
\text { RJ (1996)[12] }\end{array}$ & +++ & + & ++ & ++ & na \\
\hline $\begin{array}{l}\text { Rossing's } \\
\text { questionnaire }\end{array}$ & $\begin{array}{l}\text { Rossing, C., } \\
\text { Hansen, E. H., \& } \\
\text { Krass, I. (2003) } \\
{[16]}\end{array}$ & $?$ & na & + & na & na \\
\hline $\begin{array}{l}\text { Ngorsuraches's } \\
\text { questionnaire }\end{array}$ & $\begin{array}{l}\text { Ngorsuraches, S., } \\
\& \text { Li, S. C. (2006) } \\
\text { [17] }\end{array}$ & $?$ & na & $?$ & na & na \\
\hline $\begin{array}{l}\text { Aburuz's } \\
\text { questionnaire }\end{array}$ & $\begin{array}{l}\text { Aburuz S. et al } \\
(2011)[19]\end{array}$ & + & na & + & na & na \\
\hline $\begin{array}{l}\text { Azhar's } \\
\text { questionnaire }\end{array}$ & $\begin{array}{l}\text { Azhar, S., Hassali, } \\
\text { M. A., \& Ibrahim, } \\
\text { M. M. I. (2011) } \\
\text { [20] }\end{array}$ & + & + & + & na & na \\
\hline PABS & $\begin{array}{l}\text { Jocić, D., \& } \\
\text { Krajnović, D. } \\
\text { (2014)[21] }\end{array}$ & $?$ & na & - & na & na \\
\hline $\begin{array}{l}\text { Al-arifi's } \\
\text { questionnaire }\end{array}$ & $\begin{array}{l}\text { Al-arifi, M.N. et al } \\
(2015)[22]\end{array}$ & $?$ & na & $?$ & na & na \\
\hline $\begin{array}{l}\text { El Hajj's } \\
\text { questionnaire }\end{array}$ & $\begin{array}{l}\text { El Hajj, M. S. et al. } \\
(2016)[23]\end{array}$ & + & na & $?$ & na & na \\
\hline \multicolumn{7}{|c|}{ Specific Instruments: Disease/Health Conditions } \\
\hline $\begin{array}{l}\text { Abduelkarem's } \\
\text { questionnaire }\end{array}$ & $\begin{array}{l}\text { Abduelkarem, A. } \\
\text { R, et al (2003)[25] }\end{array}$ & $?$ & na & + & na & na \\
\hline $\begin{array}{l}\text { Wibowo's } \\
\text { questionnaire }\end{array}$ & $\begin{array}{l}\text { Wibowo, Y., et al } \\
(2015)[26]\end{array}$ & + & na & $?$ & na & na \\
\hline $\begin{array}{l}\text { El Hajj's } \\
\text { questionnaire }\end{array}$ & $\begin{array}{l}\text { El Hajj, M.S, et al } \\
(2016)[27]\end{array}$ & + & na & + & na & na \\
\hline $\begin{array}{l}\text { Scheerder's } \\
\text { scale }\end{array}$ & $\begin{array}{l}\text { Scheerder, G., De } \\
\text { Coster, I., \& Van } \\
\text { Audenhove, C. } \\
\text { (2008)[28] }\end{array}$ & + & na & $+/-$ & na & na \\
\hline $\begin{array}{l}\text { Albassam's } \\
\text { questionnaire }\end{array}$ & $\begin{array}{l}\text { Albassam, A., \& } \\
\text { Awad, A. (2018) } \\
\text { [29] }\end{array}$ & + & na & + & na & na \\
\hline $\begin{array}{l}\text { Giannetti's } \\
\text { questionnaire }\end{array}$ & $\begin{array}{l}\text { Giannetti, V., et al } \\
\text { (2018)[30] }\end{array}$ & + & na & $?$ & na & na \\
\hline
\end{tabular}




\begin{tabular}{|c|c|c|c|c|c|c|}
\hline Instruments & Author/Year & Content & $\begin{array}{l}\text { Response } \\
\text { process }\end{array}$ & $\begin{array}{l}\text { Internal } \\
\text { structure }\end{array}$ & $\begin{array}{l}\text { Other } \\
\text { variables }\end{array}$ & Consequences \\
\hline $\begin{array}{l}\text { Ashley's } \\
\text { questionnaire }\end{array}$ & $\begin{array}{l}\text { Ashley, M. J } \\
(2007)[31]\end{array}$ & $?$ & na & ++ & na & na \\
\hline $\begin{array}{l}\text { Mohamed's } \\
\text { questionnaire }\end{array}$ & $\begin{array}{l}\text { Mohamed, S. S., } \\
\text { Mahmoud, A. A., } \\
\text { \& Ali, A. A. (2014) } \\
\text { [32] }\end{array}$ & + & na & $?$ & na & na \\
\hline \multicolumn{7}{|c|}{ Specific Instruments: Services/Activities } \\
\hline $\begin{array}{l}\text { Tai's } \\
\text { instrument }\end{array}$ & $\begin{array}{l}\text { Tai, B. Et al } \\
(2016)[33]\end{array}$ & + & na & + & na & na \\
\hline $\begin{array}{l}\text { Paluck's } \\
\text { questionnaire }\end{array}$ & $\begin{array}{l}\text { Paluck, E. C.,et al } \\
(1994)[34]\end{array}$ & ++ & na & + & na & na \\
\hline $\begin{array}{l}\text { Mohamed's } \\
\text { questionnaire }\end{array}$ & $\begin{array}{l}\text { Mohamed, S. S.- } \\
\text { E., Mahmoud, A. } \\
\text { A., \& Ali, A. A. } \\
\text { (2013)[35] }\end{array}$ & + & na & + & na & na \\
\hline $\begin{array}{l}\text { Shah's } \\
\text { questionnaire }\end{array}$ & $\begin{array}{l}\text { Shah, B., \& } \\
\text { Chawla, S. (2011) } \\
\text { [36] }\end{array}$ & + & na & $?$ & na & na \\
\hline $\begin{array}{l}\text { MMAM } \\
\text { instrument }\end{array}$ & $\begin{array}{l}\text { Witry, M. J., et al } \\
(2016)[38]\end{array}$ & ++ & na & ++ & + & na \\
\hline $\begin{array}{l}\text { Perreault's } \\
\text { questionnaire }\end{array}$ & $\begin{array}{l}\text { Perreault, M. M.,et } \\
\text { al (2012)[39] }\end{array}$ & $?$ & na & + & na & na \\
\hline $\begin{array}{l}\text { Stewart's } \\
\text { questionnaire }\end{array}$ & $\begin{array}{l}\text { Stewart, D., et al } \\
(2015)[40]\end{array}$ & + & na & + & na & na \\
\hline $\begin{array}{l}\text { Guirguis's } \\
\text { questionnaire }\end{array}$ & $\begin{array}{l}\text { Guirguis, L. M., et } \\
\text { al (2018) [41] }\end{array}$ & na & na & $?$ & na & na \\
\hline $\begin{array}{l}\text { Elkalmi's } \\
\text { questionnaire }\end{array}$ & $\begin{array}{l}\text { Elkalmi, R. M.,et al } \\
(2014)[42]\end{array}$ & + & na & + & na & na \\
\hline $\begin{array}{l}\text { Taing's } \\
\text { questionnaire }\end{array}$ & $\begin{array}{l}\text { Taing, M., et al } \\
(2016)[43]\end{array}$ & + & na & + & na & na \\
\hline $\begin{array}{l}\text { Zardain's } \\
\text { questionnaire }\end{array}$ & $\begin{array}{l}\text { Zardain Tamargo, } \\
\text { E.,et al (2006) [44] }\end{array}$ & $?$ & + & ++ & na & na \\
\hline $\begin{array}{l}+++ \text { or }-=\text { strc } \\
\text { result, }+ \text { or }-=\mid \\
\text { poor methodol }\end{array}$ & $\begin{array}{l}\text { vidence positive/r } \\
\text { ed evidence positiv } \\
\text { al quality, na = no }\end{array}$ & $\begin{array}{l}\text { ative res } \\
\text { egative } \\
\text { rmation }\end{array}$ & $\begin{array}{l}++ \text { or }-=\mathrm{m} \\
\text { ult, }+/-=\mathrm{c} \\
\text { ailable }\end{array}$ & $\begin{array}{l}\text { lerate ev } \\
\text { licting e }\end{array}$ & $\begin{array}{l}\text { ce positi } \\
\text { ence,? = ul }\end{array}$ & $\begin{array}{l}\text { /negative } \\
\text { nown, due to }\end{array}$ \\
\hline
\end{tabular}

\section{Methodological quality of the included studies}


The studies were evaluated for methodological quality, as presented in Table 2. In the eight evaluated quality domains, eight studies met five or more quality criteria and the others $(n=19)$ had less than five criteria assessed. The most common failed quality criterion by the studies was not having well-defined inclusion and exclusion criteria and not assessing confounding factors. 
Table 2

Methodological quality of the included studies assessed by the Joanna Briggs Institute checklist for prevalence studies.

\begin{tabular}{|c|c|c|c|c|c|c|c|c|c|c|}
\hline Instruments & Author/Year & Q1* & Q2* & Q3* & Q4* & Q5* & Q6* & Q7* & Q8* & Score/Risk \\
\hline \multicolumn{11}{|c|}{ General Instruments } \\
\hline BPCS adapted & $\begin{array}{l}\text { Bell HM, et al. } \\
\text { (1998) [15] }\end{array}$ & $\mathrm{N}$ & $\mathrm{Y}$ & $\mathrm{Y}$ & Y & $\mathrm{N}$ & $\mathrm{N}$ & Y & Y & $\begin{array}{l}62.5 \% / \\
\text { Moderate }\end{array}$ \\
\hline $\begin{array}{l}\text { Rossing's } \\
\text { questionnaire }\end{array}$ & $\begin{array}{l}\text { Rossing C, } \\
\text { Hansen EH, } \\
\text { Krass I. } \\
(2003) \text { [16] }\end{array}$ & NC & $\mathrm{Y}$ & NC & $\mathrm{Y}$ & $\mathrm{N}$ & $\mathrm{N}$ & $\mathrm{NC}$ & Y & $\begin{array}{l}37.5 \% \text { / } \\
\text { High }\end{array}$ \\
\hline $\begin{array}{l}\text { Ngorsuraches' } \\
\text { questionnaire }\end{array}$ & $\begin{array}{l}\text { Ngorsuraches } \\
\text { S, Li SC. } \\
(2006) \text { [17] }\end{array}$ & NC & $\mathrm{Y}$ & NC & Y & $\mathrm{N}$ & $\mathrm{N}$ & $\mathrm{NC}$ & Y & $\begin{array}{l}37.5 \% \text { / } \\
\text { High }\end{array}$ \\
\hline BPCS adapted & $\begin{array}{l}\text { Hughes CM, } \\
\text { et al. }(2010) \\
\text { [18] }\end{array}$ & NC & $\mathrm{Y}$ & $\mathrm{Y}$ & $\mathrm{Y}$ & $\mathrm{Y}$ & $\mathrm{N}$ & $\mathrm{Y}$ & Y & $75 \%$ / Low \\
\hline $\begin{array}{l}\text { Aburuz's } \\
\text { questionnaire }\end{array}$ & $\begin{array}{l}\text { Aburuz S, et } \\
\text { al. (2011)[19] }\end{array}$ & $\mathrm{N}$ & $\mathrm{Y}$ & $\mathrm{Y}$ & Y & $\mathrm{N}$ & N & $\mathrm{NC}$ & $\mathrm{N}$ & $\begin{array}{l}37.5 \% \text { / } \\
\text { High }\end{array}$ \\
\hline $\begin{array}{l}\text { Azhar's } \\
\text { questionnaire }\end{array}$ & $\begin{array}{l}\text { Azhar S, } \\
\text { Hassali MA, } \\
\text { Ibrahim MMI. } \\
(2011)[20]\end{array}$ & NC & $\mathrm{Y}$ & $\mathrm{Y}$ & Y & $\mathrm{N}$ & $\mathrm{N}$ & $\mathrm{NC}$ & Y & $\begin{array}{l}50 \% \text { / } \\
\text { Moderate }\end{array}$ \\
\hline $\begin{array}{l}\text { Al-Arifi's } \\
\text { questionnaire }\end{array}$ & $\begin{array}{l}\text { Al-Arifi MN, et } \\
\text { al. (2015) [22] }\end{array}$ & $\mathrm{NC}$ & $\mathrm{Y}$ & $\mathrm{Y}$ & Y & $\mathrm{N}$ & $\mathrm{N}$ & $\mathrm{NC}$ & NC & $\begin{array}{l}37.5 \% \text { / } \\
\text { High }\end{array}$ \\
\hline $\begin{array}{l}\text { El Hajj's } \\
\text { questionnaire }\end{array}$ & $\begin{array}{l}\text { El Hajj MS, Al- } \\
\text { Saeed HS, } \\
\text { Khaja M. } \\
(2016)[23]\end{array}$ & $\mathrm{Y}$ & $\mathrm{Y}$ & NC & $\mathrm{Y}$ & $\mathrm{N}$ & $\mathrm{N}$ & $\mathrm{NC}$ & $Y$ & $\begin{array}{l}50 \% / \\
\text { Moderate }\end{array}$ \\
\hline
\end{tabular}

\section{Specific Instruments: Disease/Health Conditions}

$\begin{array}{lllllllllllll}\text { DAS adapted } & \begin{array}{l}\text { Schapansky } \\ \text { LM, Johnson } \\ \text { JA. }(2000)\end{array} & N & & & & & & & & & & \end{array}$

$\begin{array}{llllllllllll}\begin{array}{l}\text { Abduelkarem's } \\ \text { questionnaire }\end{array} & \begin{array}{l}\text { Abduelkarem } \\ \text { AR, et al. } \\ \text { (2003) [25] }\end{array} & \text { N } & \text { Y } & \text { Y } & \text { Y } & \text { N } & \text { N } & \text { N } & \text { NC } & \begin{array}{l}37.5 \% / \\ \text { High }\end{array} \\ \begin{array}{l}\text { Wibowo's } \\ \text { questionnaire }\end{array} & \begin{array}{l}\text { Wibowo Y, et } \\ \text { al. (2015)[26] }\end{array} & \text { Y } & \text { Y } & \text { NC } & \text { Y } & \text { N } & \text { N } & \text { NC } & \text { Y } & \begin{array}{l}50 \% / \\ \text { Moderate }\end{array} \\ \begin{array}{ll}\text { El Hajj's } \\ \text { questionnaire }\end{array} & \begin{array}{l}\text { El Hajj MS, et } \\ \text { al. (2016) [27] }\end{array} & \text { Y } & \text { Y } & \text { NC } & \text { Y } & \text { N } & \text { Y } & \text { NC } & \text { Y } & \begin{array}{l}62.5 \% / \\ \text { Moderate }\end{array}\end{array}$




\begin{tabular}{|c|c|c|c|c|c|c|c|c|c|c|}
\hline Instruments & Author/Year & Q1* & Q2* & Q3* & Q4* & Q5* & Q6* & Q7* & Q8* & Score/Risk \\
\hline $\begin{array}{l}\text { Scheerder's } \\
\text { scale }\end{array}$ & $\begin{array}{l}\text { Scheerder G, } \\
\text { De Coster I, } \\
\text { Van } \\
\text { Audenhove C. } \\
\text { (2008) [28] }\end{array}$ & Y & $\mathrm{Y}$ & $\mathrm{N}$ & $\mathrm{Y}$ & $\mathrm{N}$ & $\mathrm{N}$ & $\mathrm{N}$ & Y & $\begin{array}{l}50 \% / \\
\text { Moderate }\end{array}$ \\
\hline $\begin{array}{l}\text { Albassam's } \\
\text { questionnaire }\end{array}$ & $\begin{array}{l}\text { Albassam A, } \\
\text { Awad A. } \\
\text { (2018) [29] }\end{array}$ & NC & $\mathrm{Y}$ & NC & Y & $\mathrm{N}$ & Y & NC & NC & $\begin{array}{l}37.5 \% / \\
\text { High }\end{array}$ \\
\hline $\begin{array}{l}\text { Giannetti's } \\
\text { questionnaire }\end{array}$ & $\begin{array}{l}\text { Giannetti V, et } \\
\text { al. (2018) [30] }\end{array}$ & NC & $\mathrm{Y}$ & Y & Y & $\mathrm{N}$ & $\mathrm{N}$ & NC & $\mathrm{N}$ & $\begin{array}{l}37.5 \% / \\
\text { High }\end{array}$ \\
\hline $\begin{array}{l}\text { Ashley's } \\
\text { questionnaire }\end{array}$ & $\begin{array}{l}\text { Ashley MJ. } \\
\text { (2007) [31] }\end{array}$ & $\mathrm{N}$ & $\mathrm{Y}$ & Y & Y & $\mathrm{N}$ & $\mathrm{N}$ & Y & Y & $\begin{array}{l}62.5 \% / \\
\text { Moderate }\end{array}$ \\
\hline $\begin{array}{l}\text { Mohamed's } \\
\text { questionnaire }\end{array}$ & $\begin{array}{l}\text { Mohamed, } \\
\text { SS, } \\
\text { Mahmoud, } \\
\text { AA, Ali AA. } \\
\text { (2014) [32] }\end{array}$ & Y & $\mathrm{Y}$ & NC & Y & $\mathrm{N}$ & $\mathrm{N}$ & NC & $\mathrm{N}$ & $\begin{array}{l}37.5 \% / \\
\text { High }\end{array}$ \\
\hline \multicolumn{11}{|c|}{ Specific Instruments: Services/Activities } \\
\hline $\begin{array}{l}\text { Tai's } \\
\text { instrument }\end{array}$ & $\begin{array}{l}\text { Tai B, et al. } \\
(2016) \text { [33] }\end{array}$ & $\mathrm{N}$ & $\mathrm{Y}$ & $Y$ & $Y$ & $\mathrm{~N}$ & Y & NC & Y & $\begin{array}{l}62.5 \% / \\
\text { Moderate }\end{array}$ \\
\hline $\begin{array}{l}\text { Paluck's } \\
\text { questionnaire }\end{array}$ & $\begin{array}{l}\text { Paluck EC, et } \\
\text { al. (1994) [34] }\end{array}$ & NC & $\mathrm{Y}$ & $Y$ & $Y$ & $\mathrm{~N}$ & $\mathrm{~N}$ & $Y$ & Y & $\begin{array}{l}62.5 \% / \\
\text { Moderate }\end{array}$ \\
\hline $\begin{array}{l}\text { Mohamed's } \\
\text { questionnaire }\end{array}$ & $\begin{array}{l}\text { Mohamed } \\
\text { SS-E, } \\
\text { Mahmoud } \\
\text { AA, \& Ali AA. } \\
\text { (2013) [35] }\end{array}$ & $Y$ & $\mathrm{Y}$ & NC & $Y$ & $\mathrm{~N}$ & $\mathrm{~N}$ & NC & NC & $\begin{array}{l}37.5 \% / \\
\text { High }\end{array}$ \\
\hline $\begin{array}{l}\text { Shah's } \\
\text { questionnaire }\end{array}$ & $\begin{array}{l}\text { Shah B, } \\
\text { Chawla S. } \\
\text { (2011) [36] }\end{array}$ & NC & $\mathrm{Y}$ & NC & $Y$ & $\mathrm{~N}$ & $\mathrm{~N}$ & NC & Y & $\begin{array}{l}37.5 \% / \\
\text { High }\end{array}$ \\
\hline $\begin{array}{l}\text { Isenor's } \\
\text { questionnaire }\end{array}$ & $\begin{array}{l}\text { Isenor JE, et } \\
\text { al. (2018) [37] }\end{array}$ & $Y$ & $\mathrm{Y}$ & $\mathrm{NC}$ & $Y$ & $\mathrm{~N}$ & $\mathrm{~N}$ & NC & $\mathrm{N}$ & $\begin{array}{l}37.5 \% / \\
\text { High }\end{array}$ \\
\hline $\begin{array}{l}\text { Perreault's } \\
\text { questionnaire }\end{array}$ & $\begin{array}{l}\text { Perreault MM, } \\
\text { et al. (2012) } \\
\text { [39] }\end{array}$ & $\mathrm{N}$ & $Y$ & NC & $Y$ & $\mathrm{~N}$ & Y & NC & $\mathrm{NC}$ & $\begin{array}{l}37.5 \% / \\
\text { High }\end{array}$ \\
\hline $\begin{array}{l}\text { Stewart's } \\
\text { questionnaire }\end{array}$ & $\begin{array}{l}\text { Stewart D, et } \\
\text { al. (2015) [40] }\end{array}$ & $\mathrm{N}$ & $\mathrm{Y}$ & $Y$ & $Y$ & $\mathrm{~N}$ & $\mathrm{~N}$ & $Y$ & Y & $\begin{array}{l}62.5 \% / \\
\text { Moderate }\end{array}$ \\
\hline $\begin{array}{l}\text { Elkalmi's } \\
\text { questionnaire }\end{array}$ & $\begin{array}{l}\text { Elkalmi RM, } \\
\text { et al. (2014) } \\
\text { [42] }\end{array}$ & $\mathrm{N}$ & $\mathrm{Y}$ & NC & $Y$ & $\mathrm{~N}$ & $\mathrm{~N}$ & NC & Y & $\begin{array}{l}37.5 \% / \\
\text { High }\end{array}$ \\
\hline $\begin{array}{l}\text { Taing's } \\
\text { questionnaire }\end{array}$ & $\begin{array}{l}\text { Taing M, et } \\
\text { al. (2016) [43] }\end{array}$ & $\mathrm{N}$ & $Y$ & NC & $Y$ & $\mathrm{~N}$ & $\mathrm{~N}$ & NC & Y & $\begin{array}{l}37.5 \% / \\
\text { High }\end{array}$ \\
\hline
\end{tabular}




\begin{tabular}{|c|c|c|c|c|c|c|c|c|c|c|}
\hline Instruments & Author/Year & Q1* & Q2* & Q3* & Q4* & Q5* & Q6* & Q7* & Q8* & Score/Risk \\
\hline $\begin{array}{l}\text { PSOPSC } \\
\text { adapted }\end{array}$ & $\begin{array}{l}\text { Jia PL, et al. } \\
\text { (2014) [45] }\end{array}$ & $Y$ & $Y$ & $Y$ & $Y$ & $\mathrm{~N}$ & $N$ & $Y$ & $Y$ & w \\
\hline \multicolumn{11}{|c|}{ 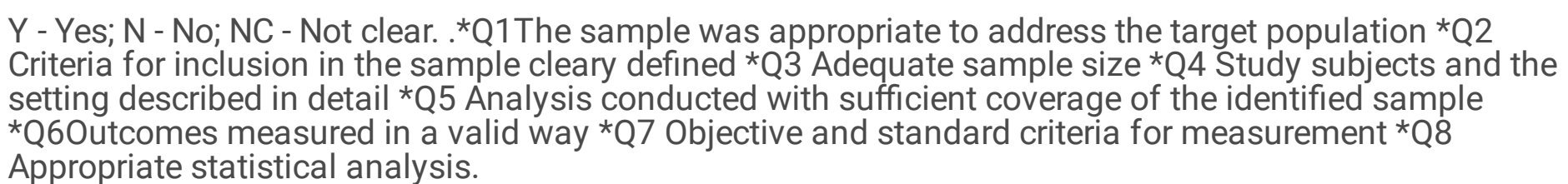 } \\
\hline
\end{tabular}

\section{Discussion}

No other systematic review seeking to identify instruments to evaluate the performance of the clinical pharmacist in different pharmaceutical practice environments was found during this study. Thirty-two studies were included, and 32 instruments were identified to be used by pharmacists acting in various pharmaceutical practice scenarios. The instruments varied in terms of type (general and specific, according to the approached constructs), methods used (from literature review to mixed methods), items, dimensions and domains, and psychometric properties (validity and reliability).

A different approach was taken in this review compared to that in other studies, in which all instruments, regardless of the practice scenario, were analyzed. In another study, in which the objective was to evaluate instruments in clinical pharmaceutical practice, the authors Alshakrah, Steinke, \& Lewis [47] limited the practice environment by selecting the instruments for the hospital environment. However, considering that clinical pharmacy covers not only hospital environments, our study sought to identify the tools for other environments, such as community and outpatient pharmacies.

In addition, the diversity of approaches to measuring pharmaceutical practice was broad for most analyzed criteria, particularly concerning the constructs and practice scenarios of interest for each study. This may be related to the different levels of knowledge and experience of pharmacists in each region of the country. This depends on the obtained training, additional qualifications in clinical pharmacy, and their individual fields of interest. For example, some pharmacists are active in the field of acute illness treatment, whereas others prefer to focus on chronic diseases, in addition to the different types of clinical services offered [48].

Regarding the construct, in the included studies, some instruments were aimed at evaluating the pharmaceutical performance in specific chronic diseases. Considering these instruments, studies that evaluated the performance of the pharmacist in the management of diabetes $[25,26,27]$, cardiovascular diseases [28], and mental illnesses [29, 31] prevailed. In some cases, the country's legislation emphasizes the necessity of involving pharmacists in the follow-up of chronic diseases [33].

Owing to this extensive approach, many of the instruments included were developed by the authors themselves as a secondary objective of the study. Therefore, 27 of them were classified as methodological and cross-sectional. In the analysis of the method, it was found that some authors did not describe the method and others classified the studies only as cross-sectional. Considering that some of these studies 
developed instruments and followed criteria of validity and reliability, these were classified as methodological and cross-sectional.

For the development of these instruments, different methodologies were used, and the literature review with the adaptation of items from other studies was the most used type for the creation of the instruments' items. Besides to this technique, the literature review combined with expert's consensus techniques that was used for the development of some instruments in this study, was also reported in another review [49].

Some studies adapted validated instruments available in the literature, including the Behavioral Pharmaceutical Care Scale (BPCS), Pharmacy Survey on Patient Safety Culture (PSOPSC), and Diabetes Attitude Scale (DAS). The BPCS instrument [15] was the most extensively examined regarding the psychometric properties and the one offering more robust statistical results, being used and mentioned in many other studies. However, this instrument is to be applied only in the community pharmaceutical practice environment, which limits its use. The advantages of utilizing validated instruments include psychometric measures like validity and reliability, allowing the comparison of different studies. However, the limitations of the use of these instruments refer to the difficulties in finding an instrument for local use and meeting the proposed objectives. Furthermore, the adapted instruments did not follow the cross-cultural adaptation methodology.

Regarding the structure and the instruments dimension, the sample size in psychometric studies is based on the number of items in the instrument and aims to provide greater assurance in their analysis and quality $[50,51]$. A sample of ten participants per item is considered acceptable, but there are studies that prove that twenty or more can significantly reduce error and inaccuracies in the solution of psychometric models, such as percentage of samples with correct factorial structure, average number of items classified incorrectly in the wrong factor, average error in eigenvalues, average error in factorial loads, the percentage of analyzes that do not converge after 250 interactions, and percentage with Heywood cases [52].

The limitation in the sample requires that initial minimum parameters of adequacy, such as factorial loads, commonality and the goodness of fit indexes are greater than in studies with larger samples. In only two of the 32 articles analyzed, the ratio between the number of participants for each item of the instrument was greater than 20: 1 and in five articles the ratio was 10: 1. However, no study has reported whether the sample size was determined and whether this fact also guided the establishment of the minimum parameters model.

As for the reliability measure, most studies used Cronbach's Alpha with acceptable values within the established criteria. This coefficient depends on the magnitude of the correlation between the items and the number of items on the instrument [53]. Many studies have criticized the use of Alpha without considering the nature and distribution of the data and the sample size, especially in samples involving more than 1000 participants $[54,55]$. The use of McDonald's Omega and Greatest Lower Bound are preferable when there is data asymmetry, even in small samples and where high alpha values do not necessarily mean greater reliability and quality of the scales or tests, because they can be the result of long scales with parallel and redundant items or generate a restriction in the construct under study [56,57].

Page 13/21 
Also, of the 32 studies analyzed, three performed test-retest reliability and did not validate the instrument's construct. Bertchold 2016 [58] questions the use of the reliability term in the test-retest, reinforcing that Pearson's correlation is a measure of association and not of reliability. Another way to clarify the reliability of an instrument and the possibility of guaranteeing its quality in different contexts is through invariance tests.

In this review, different instruments were identified to measure the performance of the clinical pharmacist, but several weaknesses were detected in the available instruments. According to the parameters and evidence criteria, few have undergone validation procedures with satisfactory results. Many authors refer to evidence from only one or two sources, such as reliability or correlation with the scores of another instrument, to support the validity of interpretations and, therefore, should be used with caution [59].

Concerning the methodological quality of the included cross-sectional studies, $55 \%$ of the studies had a high risk of bias. The JBI checklist addresses critical issues of internal and external validity that should be considered when assessing the validity of study prevalence data [60]. Therefore, the high risk of bias in these studies allows us to have more consistent and reliable conclusions from the data obtained, with important implications for data comparison.

The strengths of this study include the non- restrictions of language and time; high agreement between reviewers in the title and in the screening for inclusion of the studies; psychometric assessment of the instruments for methodological studies. As for the limitations, some studies may not have been found, as they are not indexed in the selected databases, or because they were published by institutions, foundations, or societies.

\section{Conclusion}

Thirty-two instruments which evaluated the role of the clinical pharmacist in different pharmaceutical practice scenarios, with weakness in the psychometric properties of the instruments and in the methodological quality of the studies. Also, a standardized and validated instrument that comprehensively assessed the performance of the clinical pharmacist, addressing clinical activities, was not identified for all practice environments. Thus, it is hard to establish the main clinical activities performed by pharmacists in their pharmaceutical practice environments and to propose training actions to improve professional practice. Despite the large number of instruments available and considered validated by the authors, it is questioned to what extent the validity indicators presented in the different studies really show the validation status.

\section{Abbreviations}

BPCS: Behavioral Pharmaceutical Care Scale

PSOPSC: Pharmacy Survey on Patient Safety Culture

DAS: Diabetes Attitude Scale 


\section{Declarations}

\section{Ethics approval and consent to participate:}

'Not applicable'.

\section{Consent for publication:}

'Not applicable'.

\section{Availability of data and materials:}

"All data generated or analysed during this study are included in this published article [and its supplementary information files]".

\section{Competing interests:}

"The authors declare that they have no competing interests".

\section{Funding:}

'Not applicable'.

\section{Authors' contributions:}

"DPCQ participated in the selection of studies and data extraction. CCP, TML, MN, FR was a major contributor in writing the manuscript. All authors read and approved the final manuscript".

\section{Acknowledgements:}

'Not applicable'.

\section{References}

1. Cipolle RJ, Strand L, Morley P. Pharmaceutical care practice: The patient - centered approach to medication management. 3 ed. New York: McGraw-Hill Medical; 2012.

2. Buss $\mathrm{VH}$, Shield $A$, Kosari $S$, Naunton $M$. The impact of clinical services provided by community pharmacies on the Australian healthcare system: a review of the literature. J Pharm Policy Pract. 2018;11(22). 
3. Hansen RA, Roth MT, Brouwer ES, Herndon S, Christensen DB. Medication therapy management services in North Carolina community pharmacies: current practice patterns and projected demand. J Am Pharm Assoc. 2006;46(6):700-706.

4. Greißing C, Kössler K, Freyer J, Hüter L, Buchal P, Schiek S, et al. The status of the performance of medication reviews in German community pharmacies and assessment of the practical performance. Int J Clin Pharm. 2016;38(6):1425-1435. doi:10.1007/s11096-016-0381-7

5. Dalton K, Byrne S. Role of the pharmacist in reducing healthcare costs: current insight. Integr Pharm Res Pract. 2017;6:37-46.

6. Keszei A, Novak M, Streiner DL. Introduction to health measurement scales. J Psychosom Res. 2010;68(4):319-323.

7. American Educational Research Association, American Psychological Association \& National Council on Measurement in Education. (1999). Standards for educational and psychological testing. Washington: American Psychological Association.

8. Kane, M T. Explicating validity. Assessment in Education: Principles, Policy \& Practice. 2016; 23:2, 198211, doi: 10.1080/0969594X.2015.1060192

9. Booth A, Clarke M, Dooley G, Ghersi D, Moher D, Petticrew M, et al. The nuts and bolts of PROSPERO: an international prospective register of systematic reviews. Syst Rev. 2012;1:2. doi:10.1186/2046-4053-1-2

10. Moher D, Shamseer L, Clarke M, Ghersi D, Liberati A, Petticrew M, et al. Preferred reporting items for systematic review and meta-analysis protocols (PRISMA-P) 2015 statement. Syst Rev. 2015;4:1. doi:10.1186/2046-4053-4-1

11. Joanna Briggs Institute. Meta-analysis of statistics: assessment and review instrument (JBI-MAStARI). Adelaide: The Joanna Briggs Institute; 2006.

12. Joanna Briggs Institute. The Joanna Briggs Institute Critical Appraisal Tools for Use in JBI Systematic Reviews Checklist for Analytical Cross Sectional Studies. North Adelaide, Australia: The Joanna Briggs Institute; 2017.

13. Borsboom D, Mellenbergh GJ. and van Heerden J. The concept of validity. Psychological Review. 2004, 111(4): 1061-1071.

14. Furlan AD, Pennick V, Bombardier C, van Tulder M. 2009 updated method guidelines for systematic reviews in the Cochrane Back review group. Spine (Phila Pa 1976). 2009;34:1929-41. https://doi.org/10.1097/BRS. 0b013e3181b1c99f

15. Odedina FT, Segal RJ. Behavioral pharmaceutical care scale for measuring pharmacists' activities. Am J Health Syst Pharm. 1996;53(8):855-65.

16. Bell HM, McElnay JC, Hughes CM, Woods A. Provision of pharmaceutical care by community pharmacists in Northern Ireland. Am J Health Syst Pharm. 1998;55(19):2009-2013. 
17. Rossing C, Hansen EH, Krass I. The provision of pharmaceutical care in Denmark: A cross-sectional survey. J Clin Pharm Ther. 2003;28(4):311-318. https://doi.org/10.1046/j.1365-2710.2003.00498.x

18. Ngorsuraches S, Li SC. Thai pharmacists' understanding, attitudes, and perceived barriers related to providing pharmaceutical care. Am J Health Syst Pharm. 2006;63(21):2144-2150. https://doi.org/10.2146/ajhp060054

19. Hughes CM, Hawwa AF, Scullin C, Anderson C, Bernsten CB, Bjornsdottir I. Provision of pharmaceutical care by community pharmacists: a comparison across Europe. Pharm World Sci. 2010;32(4):472-487. https://doi.org/10.1007/s11096-010-9393-x

20. Aburuz S, Al-Ghazawi M, Snyder A. Pharmaceutical care in a community-based practice setting in Jordan: where are we now with our attitudes and perceived barriers? Int J Pharm Pract. 2012; 20(2):7179.

21. Azhar S, Hassali MA, Ibrahim MMI. Perceptions of hospital pharmacist's role in Pakistan's healthcare system: A cross-sectional survey. Trop J Pharm Res. 2011;10(1):11-17.

22. Jocić D, Krajnović D. Development and initial validation of a scale to measure attitudes and beliefs of pharmacists toward their work with patients. Vojnosanitetski Pregled. 2014;71(4):373-382. https://doi.org/10.2298/VSP120314051J

23. Al-Arifi MN, Dhawailie AA, Aldohyan M, Wajid S, Babelgaith SD, Rajab S, et al. A survey on pharmacist opinion about pharmaceutical care in Saudi Arabia. Asian J Pharm. 2015;9(4):277-282.

24. El Hajj MS, AL-Saeed HS, Khaja M. Qatar pharmacists' understanding, attitudes, practice and perceived barriers related to providing pharmaceutical care. Int J Clin Pharm. 2016;38(2):330-343.

25. Schapansky LM, Johnson JA. Pharmacists' attitudes toward diabetes. J Am Pharm Assoc (Wash). 2000;40(3):371-377. https://doi.org/10.1016/S1086-5802(16)31085-3

26. Abduelkarem AR, Sackville MA, Morgan RM, Sackville MP, Hildreth AJ. Views and practices of community pharmacists regarding services for people with type 2 diabetes. Int J Pharm Pract. 2003;11(3):161-168.

27. Wibowo Y, Parsons R, Sunderland B, Hughes J. Evaluation of community pharmacy-based services for type-2 diabetes in an Indonesian setting: pharmacist survey. Int J Clin Pharm. 2015;37(5):873-882. https://doi.org/10.1007/s11096-015-0135-y

28. El Hajj MS, Mahfoud ZR, Al Suwaidi J, Alkhiyami D, Alasmar AR. Role of pharmacist in cardiovascular disease-related health promotion and in hypertension and dyslipidemia management: a cross-sectional study in the State of Qatar. J Eval Clin Pract. 2016;22(3):329-340.

29. Scheerder G, De Coster I, Van Audenhove C. Pharmacists' role in depression care: a survey of attitudes, current practices, and barriers. Psychiatr Serv. 2008;59(10):1155-1160. 
https://doi.org/10.1176/ps.2008.59.10.1155

30. Albassam A, Awad, A. Community pharmacists' services for women during pregnancy and breast feeding in Kuwait: A cross-sectional study. BMJ Open. 2018; 8(1): e018980.

https://doi.org/10.1136/bmjopen-2017-018980

31. Giannetti V, Caley, CF, Kamal KM, Covvey JR, McKee J, Wells BG, et al. Community pharmacists and mental illness: a survey of service provision, stigma, attitudes and beliefs. Int J Clin Pharm. 2018; 40(5):1096-1105. https://doi.org/10.1007/s11096-018-0619-7

32. Ashley MJ, Victor JC, Brewster J. Pharmacists' attitudes, role perceptions and interventions regarding smoking cessation: Findings from four Canadian provinces. Chronic Dis Can. 2007;28(1-2):20-28.

33. Mohamed SS, Mahmoud AA, Ali AA. The role of Sudanese community pharmacists in patients' selfcare. Int J Clin Pharm. 2014;36(2):412-419. https://doi.org/10.1007/s11096-013-9911-8

34. Tai B-WB, Hata M, Wu S, Frausto S, Law AV. Prediction of pharmacist intention to provide medication disposal education using the theory of planned behaviour. J Eval Clin Pract. 2016;22(5):653-661. https://doi.org/10.1111/jep.12511

35. Paluck EC, Stratton TP, Eni GO. Community pharmacists' participation in health education and disease prevention activities. J Public Health. 1994;85(6):389-92.

36. Mohamed SS-E, Mahmoud AA, Ali AA. Involvement of Sudanese community pharmacists in public health activities. Int J Clin Pharm. 2013;35(3):393-400. https://doi.org/10.1007/s11096-012-9745-9

37. Shah B, Chawla S. A needs assessment for development and provision of medication therapy management services in New York City. J Pharm Pract. 2011;24(3):339-344. https://doi.org/10.1177/0897190010396584

38. Isenor JE, Slayter KL, Halperin DM, McNeil SA, Bowles SK. Pharmacists' immunization experiences, beliefs, and attitudes in New Brunswick, Canada. Pharm Pract. 2018;16(4): 1310. https://doi.org/10.18549/PharmPract.2018.04.1310

39. Witry MJ, Wesely PM, Goedken AM, Ernst EJ, Sorofman BA, Doucette WR. Development of a medication monitoring attitude measure using a mixed methods item development process. Int J Pharm Pract. 2016;24(1):49-59. https://doi.org/10.1111/ijpp.12185

40. Perreault MM, Thiboutot Z, Burry LD, Rose L, Kanji S, Leblanc JM. Canadian survey of critical care pharmacists' views and involvement in clinical research. Ann Pharmacother. 2012;46(9):1167-1173. https://doi.org/10.1345/aph.1R180

41. Stewart D, Al Hail M, Abdul Rouf PV, El Kassem W, Diack L, Thomas B, et al. Building hospital pharmacy practice research capacity in Qatar: a cross-sectional survey of hospital pharmacists. Int J Clin Pharm. 2015;37(3):511-521. https://doi.org/10.1007/s11096-015-0087-2

Page $18 / 21$ 
42. Guirguis LM, Hughes CA, Makowsky MJ, Sadowski CA, Schindel TJ, Yuksel N, et al. Development and validation of a survey instrument to measure factors that influence pharmacist adoption of prescribing in Alberta, Canada. Pharm Pract. 2018; 16(1): 1068.

https://doi.org/10.18549/PharmPract.2018.01.1068

43. Elkalmi RM, Hassali MA, Ibrahim MIM, Jamshed SQ, Al-Lela OQB. Community pharmacists' attitudes, perceptions, and barriers toward adverse drug reaction reporting in Malaysia: a quantitative insight. $J$ Patient Saf. 2014;10(2):81-87.

44. Taing M-W, Ford PJ, Gartner CE, Freeman CR. Describing the role of Australian community pharmacists in oral healthcare. Int J Pharm Pract. 2016;24(4):237-246. https://doi.org/10.1111/ijpp.12241

45. Zardain TE, del Valle Gomez M. del O, Lopez Gonzalez ML, Garcia Cueto, E. Validation of a questionnaire for measuring psychosocial factors associated with pharmaceutical care in Spanish community pharmacists. Psicothema. 2006;18(4):778-784.

46. Jia PL, Zhang LH, Zhang MM, Zhang LL, Zhang C, Qin SF. Safety culture in a pharmacy setting using a pharmacy survey on patient safety culture: a cross-sectional study in China. BMJ Open.

2014;4(6):e004904. https://doi.org/10.1136/bmjopen-2014-004904

47. Alshakrah MA, Steinke DT, Lewis PJ. Patient prioritization for pharmaceutical care in hospital: A systematic review of assessment tools. Res Social Adm Pharm. 2019;15(6):767-779.

48. Hampson N. Getting started with clinical pharmacists in general practice. Prescriber, 2018;29(3):25-28.

49. Lima T de M, Aguiar PM, Storpirtis S. Evaluation of quality indicator instruments for pharmaceutical care services: A systematic review and psychometric properties analysis. Res Social Adm Pharm. 2018;14(5):405-412.

50. Hair JF. Multivariate data analysis. 7th ed. NJ: Pearson Prentice Hall; 2010.

51. Tabachnick BG, Fidell LS. Using multivariate statistics. 6th ed. Boston: Pearson Education; 2013

52. Costello $A B$, Osborne JW. Best practices in exploratory factor analysis: four recommendations for getting the most from your analysis. Pract Assessment, Res Eval. 2005;10(7):1-9.

53. Streiner DL, Norman GR, Cairney J. Health measurement scales : a practical guide to their development and use. 5th ed. Oxford: Oxford University Press; 2015. 53.

54. Sijtsma K. On the use, the misuse, and the very limited usefulness of Cronbach's alpha. Psychometrika. 2009;74:107-20. https://doi.org/10.1007/ s11336-008-9101-0.

55. Zinbarg RE, Revelle W, Yovel I, Li W. Cronbach's a, Revelle's $\beta$, and Mcdonald's $\omega H$ : their relations with each other and two alternative conceptualizations of reliability. Psychometrika. 2005;70:123-33. https://doi. org/10.1007/s11336-003-0974-7.

56. Trizano-Hermosilla I, Alvarado JM. Best alternatives to Cronbach's alpha reliability in realistic conditions: congeneric and asymmetrical measurements. Front Psychol. 2016;7:769. https://doi.org/10.3389/fpsyg.2016. 00769. 
57. Panayides $\mathrm{P}$, Walker MJ. Evaluating the psychometric properties of the foreign language classroom anxiety scale for Cypriot senior high school EFL students: the Rasch measurement approach. Eur J Psychol. 2013;9:493-516.

58. Berchtold A. Test-retest: agreement or reliability? Methodol Innov. 2016;9: 205979911667287. https://doi.org/10.1177/2059799116672875.

59. Cook DA, Beckman TJ. Current concepts in validity and reliability for psychometric instruments: theory and application. Am J Med. 2006;119:166.e7-16. [PubMed]

60. Munn Z, Moola S, Riitano D, Lisy K. The development of a critical appraisal tool for use in systematic reviews addressing questions of prevalence. Int J Health Policy Manag. 2014;3(3):123-128. Published 2014 Aug 13. doi:10.15171/ijhpm.2014.71

\section{Figures}

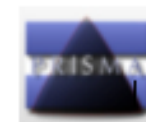

PRISMA 2009 Flow Diagram

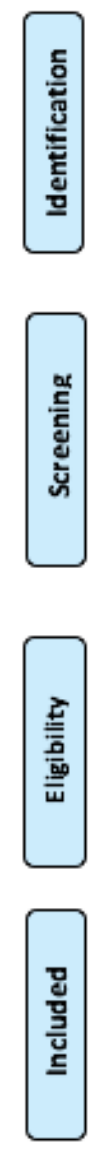

Records identified through PubMed

(1.727) and Scopus (2.369)

database searching ( $n=4.096$ )

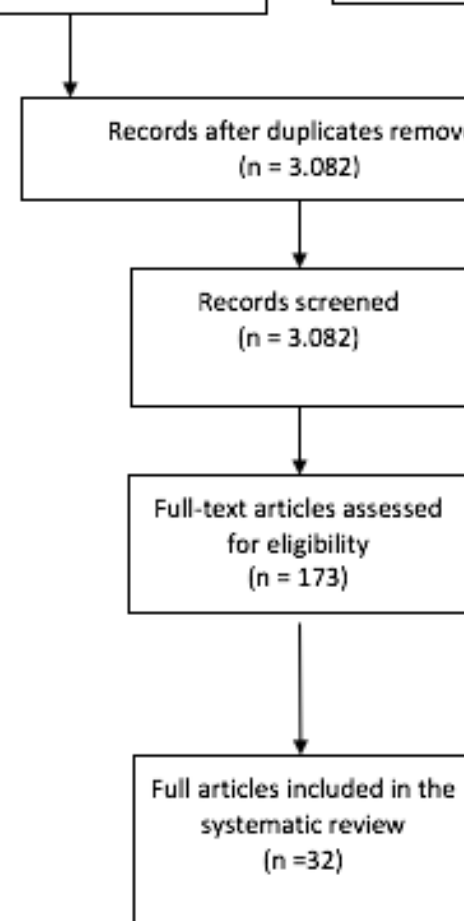

Additional records through references lists $(n=4)$

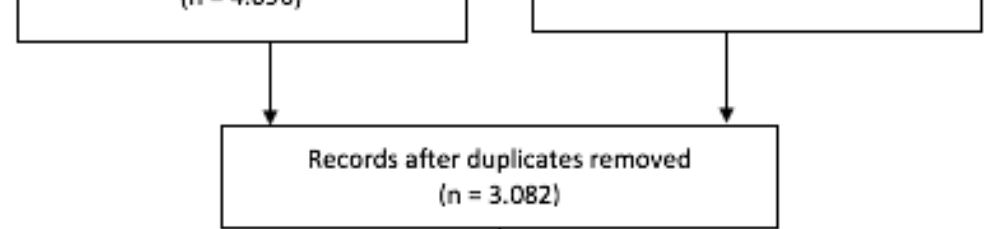

Records excluded based on titles and abstracts ( $n=2.909)$

Full-text articles excluded, with reasons $(n=141)$ Perception of other professionals: 2 No instruments: 1 Full text not found: 9 Questionnaires which were not validated:96 Other results: 33

\section{Figure 1}

Flow diagram of study searching and selection process. 


\section{Supplementary Files}

This is a list of supplementary files associated with this preprint. Click to download.

- Additionalfile1.doc

- Additionalfile2.docx

- Additionalfile3.doc

- Additionalfile4.docx

- Additionalfile5.docx 